\title{
UNIFORM ESTIMATES OF A TRIGONOMETRIC INTEGRAL \\ BY
}

DANIEL WATERMAN (SYRACUSE, NEW YORK)

FOR ANTONI ZYGMUND WITH RESPECT AND GRATITUDE

Let us suppose that $f$ is a real function on the circle group $T$ and $S_{n}$ is the $n$th partial sum of its Fourier series. A basic tool in the study of the convergence of Fourier series is the fact that, for any $\delta>0$,

$$
S_{n}(x)-f(x)=\frac{1}{\pi} \int_{0}^{\delta}(f(x+t)+f(x-t)-2 f(x)) \frac{\sin n t}{t} d t+o(1),
$$

uniformly in $x$, on any interval where $f$ is bounded [5, p. 55]. In this paper we will make uniform estimates of integrals of this form for continuous $f$ with $\delta$ fixed and with $\delta$ varying with $n$.

The estimates we supply here have been used by us [4] to furnish an alternative demonstration of the result of Baernstein and Waterman [1] establishing the necessary and sufficient condition for the Fourier series of $f \circ g$ to converge uniformly for every homeomorphism $g$ of $T$ with itself. The first estimate of this type was used in the argument by which Goffman and Waterman [2] solved the analogous problem for everywhere convergence.

Let $\omega(f, \delta)$ denote the modulus of continuity of $f$. If $h=h(x, t)$ is a function on $T^{2}$, set

$$
\bar{\omega}(\delta)=\bar{\omega}(h, \delta)=\sup \left\{\left|h(x, t)-h\left(x, t^{\prime}\right)\right||| t-t^{\prime} \mid<\delta, x \in T\right\} .
$$

In the case

$$
h(x, t)=f(x+t)+f(x-t)-2 f(x)
$$

it is clear that $\bar{\omega}(h, \delta) \leq 2 \omega(f, \delta)$.

We intruduce the notation

$$
\sum(h, k, n, x, \theta)=\sum_{i=1}^{k} i^{-1}[h(x, 2 i \pi / n+\theta)-h(x,(2 i-1) \pi / n+\theta)] .
$$


THEOREM. Let $h(x, t)$ be a function on $T^{2}$ continuous in $t$ uniformly with respect to $x$, and with $h(x, 0)=0$ for all $x$.

(A) There is $\theta(x, n) \in(0, \pi / n)$ such that for any sequence of positive integers $\left\{k_{n}\right\}$ with $\bar{\omega}(\pi / n) \log \left(n / k_{n}\right)=o(1)$,

$$
\int_{0}^{\delta} h(x, t) \frac{\sin n t}{t} d t=\frac{1}{\pi} \sum\left(h, k_{n}, n, x, \theta(x, n)\right)+o(1)
$$

uniformly in $x$.

(B) Let $\left\{k_{n}\right\}$ be an increasing sequence of positive integers. There is a sequence $\left\{\varepsilon_{n}\right\}, 0<\varepsilon_{n}<\pi / n$, such that for any $h(x, t)$ there is $\theta(x, n) \in$ $\left(0, \pi / n-\varepsilon_{n}\right)$ such that

$$
\int_{0}^{\left(2 k_{n}+1\right) \pi / n} h(x, t) \frac{\sin n t}{t} d t=\frac{1}{\pi} \sum\left(h, k_{n}, n, x, \theta(x, n)\right)+o(1)
$$

uniformly in $x$. The choice $\varepsilon_{n}=o\left[1 / n \sqrt{\log k_{n}}\right]$ suffices for all $h$.

Suppose $I$ denotes an interval $[a, b]$. By $f(I)$ we mean $f(b)-f(a)$. Applying (A) to the particular $h$ discussed above, we obtain the

CoROLlary. If $f$ is a continuous function on $T$, then there is $\theta(x, n) \in$ $\left(0, \pi / n-\varepsilon_{n}\right)$ such that for any sequence of positive integers $\left\{k_{n}\right\}$ with $\omega(f, \pi / n) \log \left(n / k_{n}\right)=o(1)$,

$$
S_{n}(x)-f(x)=\frac{1}{\pi^{2}}\left[\sum_{i=1}^{k_{n}} i^{-1} f\left(I_{n i}\right)-\sum_{i=1}^{k_{n}} i^{-1} f\left(J_{n i}\right)\right]+o(1)
$$

uniformly in $x$, where

$$
\begin{aligned}
I_{n i} & =[x+(2 i-1) \pi / n+\theta(x, n), x+2 i \pi / n+\theta(x, n)], \\
J_{n i} & =[x-2 i \pi / n-\theta(x, n), x-(2 i-1) \pi / n-\theta(x, n)] .
\end{aligned}
$$

The methods employed in the proofs of these results are related to those used by Salem in his work on uniform convergence [3].

Writing

$$
\int_{0}^{\delta} h(x, t) \frac{\sin n t}{t} d t=\int_{0}^{\pi / n} \cdots+\int_{\pi / n}^{\delta} \cdots
$$

we have

$$
\left|\int_{0}^{\pi / n} \cdots\right| \leq \varpi(h, \pi / n) \int_{0}^{\pi}\left|\frac{\sin t}{t}\right| d t=o(1)
$$


as $n \rightarrow \infty$ uniformly in $x$. Considering the other integral,

$$
\int_{\pi / n}^{\delta} \ldots=\sum_{k=1}^{N} \int_{k \pi / n}^{(k+1) \pi / n} \cdots+\int_{(N+1) \pi / n}^{\delta} \cdots ;
$$

here $N+1$ is an odd integer such that $0 \leq \delta-(N+1) \pi / n<2 \pi / n$. Then

$$
\left|\int_{(N+1) \pi / n}^{\delta} \cdots\right|<C\|h\| / n
$$

uniformly in $x$, where $\|\cdot\|$ denotes the sup norm. We have

$$
\begin{aligned}
\sum_{k=1}^{N} \int_{k \pi / n}^{(k+1) \pi / n} h(x, t) \frac{\sin n t}{t} d t & =\int_{0}^{\pi} \sum_{k=1}^{N}(-1)^{k} h(x,(t+k \pi) / n) \frac{\sin t}{t+k \pi} d t \\
& =2 \sum_{k=1}^{N}(-1)^{k} \frac{h(x,(\bar{t}+k \pi) / n)}{\bar{t}+k \pi}
\end{aligned}
$$

for some $\bar{t} \in(0, \pi)$. Considering two consecutive terms of this sum, $k$ odd,

$$
\begin{aligned}
& -\frac{h(x,(\bar{t}+k \pi / n)}{\bar{t}+k \pi}+\frac{h(x,(\bar{t}+(k+1) \pi) / n)}{\bar{t}+(k+1) \pi} \\
& =\frac{-h(x,(\bar{t}+k \pi) / n)+h(x,(\bar{t}+(k+1) \pi) / n)}{\bar{t}+(k+1) \pi} \\
& +h(x,(\bar{t}+k \pi) / n)\left[\frac{1}{\bar{t}+(k+1) \pi}-\frac{1}{\bar{t}+k \pi}\right]
\end{aligned}
$$

and the absolute value of the last term is less than

$$
\frac{\bar{\omega}((k+1) \pi / n)}{k(k+1) \pi} \text {. }
$$

The sum of the absolute values of these terms from $k=1$ to $q$ is less than $\bar{\omega}((q+1) \pi / n) / \pi$ and the sum from $q+1$ to $N$ is less than $\|h\| /(q+1) \pi$. Choosing $q \sim \sqrt{n}$, the sum of all these terms is seen to be $o(1)$ as $n \rightarrow \infty$ uniformly in $x$. If in the terms of the form

$$
\frac{-h(x,(\bar{t}+k \pi) / n)+h(x,(\bar{t}+(k+1) \pi) / n)}{\bar{t}+(k+1) \pi}
$$

we replace the denominators by $(k+1) \pi$, observing that

$$
|1 /(\bar{t}+(k+1) \pi)-1 /(k+1) \pi|<1 /(k+1)^{2} \pi,
$$


we change the sum of these terms by $O(\bar{\omega}(\pi / n))$. Thus

$$
\begin{aligned}
& \int_{\pi / n}^{\delta} h(x, t) \frac{\sin n t}{t} d t= o(1)+\frac{2}{\pi}\left[\frac{h(x,(\bar{t}+2 \pi) / n)-h(x,(\bar{t}+\pi) / n)}{2}+\ldots\right. \\
&\left.+\frac{h(x,(\bar{t}+N \pi) / n)-h(x,(\bar{t}+(N-1) \pi) / n)}{N}\right] \\
&= o(1)+\frac{1}{\pi}\left[\sum_{i=1}^{N / 2} i^{-1}(h(x,(\bar{t}+2 i \pi) / n)\right. \\
&-h(x,(\bar{t}+(2 i-1) \pi) / n))] .
\end{aligned}
$$

Writing $\sum_{i=1}^{N / 2} \ldots=\sum_{i=1}^{k_{n}} \ldots+\sum_{k_{n}+1}^{N / 2} \ldots$, we note that

$$
\left|\sum_{k_{n}+1}^{N / 2} \ldots\right|=O\left(\bar{\omega}(\pi / n) \log \left(n / k_{n}\right)\right)=o(1)
$$

under our hypotheses, which establishes part (A) of our theorem.

Turning now to part (B), we have

$$
\int_{0}^{\left(2 k_{n}+1\right) \pi / n} h(x, t) \frac{\sin n t}{t} d t=\int_{0}^{\pi / n} \cdots+\sum_{i=1}^{2 k_{n}} \int_{i \pi / n}^{(i+1) \pi / n} \cdots
$$

and the first integral on the right is $o(1)$ as before. As for the rest, for small $\eta_{n} \in(0, \pi)$, we have

$$
\begin{array}{r}
\int_{0}^{\pi} \sin t \sum_{i=1}^{2 k_{n}}(-1)^{i} \frac{h(x,(t+i \pi) / n)}{t+i \pi} d t=\int_{0}^{\pi-\eta_{n}} \ldots+\int_{\pi-\eta_{n}}^{\pi} \ldots \\
=\left(1+\cos \eta_{n}\right) \sum_{i=1}^{2 k_{n}}(-1)^{i} \frac{h\left(x, \theta_{n}+i \pi / n\right)}{n \theta_{n}+i \pi}+R
\end{array}
$$

where $\theta_{n} \in\left(0,\left(\pi-\eta_{n}\right) / n\right)$. Then

$$
|R| \leq \eta_{n}^{2} \frac{1}{\pi} \bar{\omega}\left(\left(2 k_{n}+1\right) \pi / n\right) \sum_{i=1}^{2 k_{n}} i^{-1}=o(1)
$$

uniformly in $x$ as $n \rightarrow \infty$ if $\eta_{n}=o\left(\left(\bar{\omega}\left(\left(2 k_{n}+1\right) \pi / n\right) \log k_{n}\right)^{-1 / 2}\right)$. The sum is treated as in the previous case, yielding for the right hand side,

$$
\left(1+\cos \eta_{n}\right) \frac{1}{\pi} \sum_{i=1}^{k_{n}}(2 i)^{-1}\left[h\left(x, \theta_{n}+2 i \pi / n\right)-h\left(x, \theta_{n}+(2 i-1) \pi / n\right)\right]+o(1) .
$$


Replacing $1+\cos \eta_{n}$ by 2 , we introduce an error bounded by

$$
\left(1-\cos \eta_{n}\right) \frac{1}{2 \pi} \bar{\omega}(\pi / n) \sum_{i=1}^{k_{n}} i^{-1} \leq C \eta_{n}^{2} \bar{\omega}(\pi / n) \log k_{n}=o(1) .
$$

if $\eta_{n}$ is subject to the condition above. Part (B) then follows with $\theta(x, n) \in$ $\left(0, \pi / n-\varepsilon_{n}\right)$ where $\varepsilon_{n}=\eta_{n} / n$. Note that $\eta_{n}=o\left[1 / \sqrt{\log k_{n}}\right]$ or, equivalently, $\varepsilon_{n}=o\left[1 / n \sqrt{\log k_{n}}\right]$ suffices for our estimates and is independent of $h$.

\section{REFERENCES}

[1] A. Baernstein and D. Waterman, Functions whose Fourier series converge uniformly for every change of variable, Indiana Univ. Math. J. 22 (1972), 569-576.

[2] C. Goff $\mathrm{m}$ an and D. W at er m a n, Functions whose Fourier series converge for every change of variable, Proc. Amer. Math. Soc. 19 (1968), 80-86.

[3] R. S ale m, Essais sur les séries trigonométriques, Act. Sci. Ind. 862, Hermann, Paris 1940.

[4] D. W a te $\mathrm{m}$ a n, Functions whose Fourier series converge uniformly for every change of variable II, Indian J. Math. 25 (1983), 257-264.

[5] A. Zygmund, Trigonometric Series, Vol. I, Cambridge University Press, 1959.

DEPARTMENT OF MATHEMATICS

SYRACUSE UNIVERSITY

SYRACUSE, NEW YORK 13244-1150, U.S.A.

Reçu par la Rédaction le 14.5.1990 